\title{
A Review on: "Recent Updates on Poly-Adp-Ribosylation (Parylation) in Physiology and Pathology"
}

\author{
Pritam Paramguru Mahapatra ${ }^{1}$, Manaswini Giri ${ }^{1}$, \\ Vellore Institute of Technology, Tamil Nadu, India
}

\begin{abstract}
One of the most common cellular responses to DNA breakage is the covalent post-translational modification of the nuclear proteins with poly ADP ribose from the NAD+ as a precursor, which is mostly catalyzed by the poly-ADP-ribose polymerase1(PARP1). Recently, it has been seen, poly ADP ribose (PAR) formation has been catalyzed by several polypeptides. Moreover, the process of poly ADP Ribosylation
\end{abstract}

(PARylation) is involved in physiology and pathology phenomena. The physiological function includes the DNA-base deletion, DNA impairment signalling, etc. Whereas the pathological effects are mediated through the over-activity of PARP1 which can deplete NAD+. The latter effect undergoes the pathogenesis of a wide range of diseases with including organs blood supply, heart failure, liver problem, diabetes.

\section{Graphical Abstract}

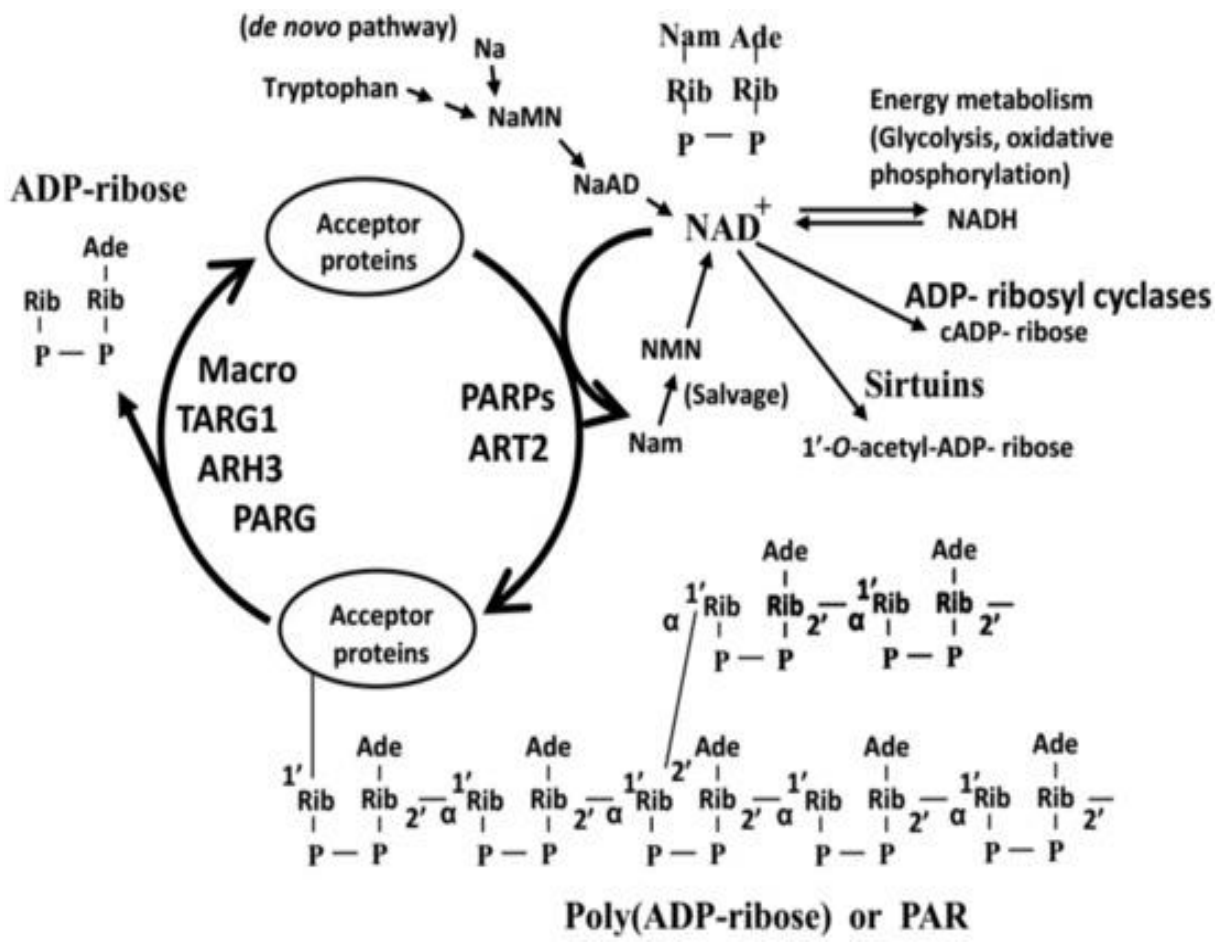

Fig 1:- Cycle of formation of poly ADP ribosylation

Keywords:- NAD+, PARP, ADPr, Post Translational Modification, DNA base editing, DNA damage signaling, ARTs.

\section{INTRODUCTION}

The notable examples of how living species are adapting and surviving in response to the variations in nature and the environment can be studied through evolution. The poly-ADP-ribose (PAR) discovery has established a novel field of science in motion, the field of poly-ADP-ribosyl transferases (PARP), and ADP Ribosylation. These adaptations are thus induced by the cascades of the molecular events that are involved such as qualitative and quantitative variations in the basic, cellular macromolecules such as proteins, nucleic acid and lipids. The most common tool to induce the quick alteration in the cellular environment is the post-transitional modification (PTM) of proteins by the addition of chemicals. Thus, PARylation is a highly conserved PTM system, the addition of ADP ribose (ADP) groups from the nicotinamide and adenine dinucleotide (NAD+) to proteins. The process of PARylation can not only happen on proteins but also macromolecules like DNA or small chemical groups. Many recent observations show that the viral genomes have been evolved from the genetic tools that enable them to modulate 
ADP Ribosylation signaling of the infected cells. In higher organisms PARylation seems to be particularly prominent. The process of ADP Ribosylation is a widespread modification that helps in controlling a vast number of cellular processes which includes DNA-damage repair, transcription, cell cycle progression, cell division, unfolded protein response, aging, nitrogen fixation, microbial pathogenicity, cell death, and many others. This field has prolonged to include the further regulation of chromatin structure, gene expression, and RNA processing in a wide range of biological systems, including reproduction, development, aging, stem cells, inflammation, metabolism, and cancers. In the physiological process the reactions are catalyzed by a cluster of enzymes, namely ADP ribosyl transferases (ARTs) which transfer single and multiple ADP ribose moieties from NAD+ to their specific targets, includes various proteins, nucleotides, antibiotics and number of small molecules. Thus, the process of PARylation can be described under physiological and pathological conditions.

\section{PHYSIOLOGICAL CONDITIONS}

\section{Par Metabolism}

Poly-ADP-ribosylation occurs in almost all mammalian cells. It stimulates several cellular responses such as DNA damage, post-transcriptional modification, telomere maintenance, etc. DNA damage induced by ionizing radiation [4]. PRAP-1, PARylation polymerase is the enzyme which catalyzes the polymerization reaction. By DNA strand breaks PRAP-1 is activated enzymatically. PRAP-1 catalyzes the reaction from the oxidized form of NAD+ by break the glycosidic bond between nicotinamide and ribose and produce ADP-ribose [4]. The target protein (acceptor) adds covalently with the ADP-ribose subunit, via an ester bond. The protein is mostly Glutamate, aspartate, and carboxyterminal lysin residue. Polymerization result polymer chain of ADP-ribose subunit, each chain having approximately 200ADP-ribose unit.

The target acceptor protein such as p53, both the subunit of NF-kB (nuclear factor kappa B) DNA ligase, DNA-polymerase, DNA-topoisomerase, histone, but most prominent acceptor protein is PRAP-1 itself. Poly-ADPribose glycohydrolase (PRAG) hydrolyzes the ADP-ribose polymers to free ADP-ribose. The enzyme cleaves the glycosidic bond by endoglycosidase activity along with exoglycosidic activity. The breakdown produces free PAR and ADP-ribose monomer [4].

\section{$>$ Poly-Adp-Ribosylation in Centromere Protein}

Centromere form the primary constriction of the mammalian metaphase chromosome. During miotic cell, division centromere is the primary site of formation of the microtubule, which the kinetochore and regulate cell division [3]. Centromere provides essential functions such as structural organization, cell division, movement, checkpoint control. All the cellular activity the centromerespecific proteins play a vital role. Centromere specific protein can be two types; Constitutive and Transit protein. Constitutive protein such as CENP-A, CENP-B, CENP-C,
CENP-G \& CENP-H. And Transit protein such as CENPE, CENP-F, INCENP, Survivin, MCAK, ZWINT-1, ZW10, MAD-2, MAD-1, BUB1, BUBR1. PARP-1 is the enzyme that catalyzes the formation of PAR units makes the acceptor protein more negatively charge, thus altering their structure, function, and binding property to DNA. PRAP-1 associate with several centromere-specific protein CENPA, CENP- and the spindle checkpoint protein BUB3 and induce the DNA damage [6]. PTM of centromere associated protein is regulating kinetochore assembly and centromere activity. Phosphorylation of several protein CENP-A (CENP-B, 3F $3 / 2$ epitope, and topoisomerase IIIA has been reported. PARylation of protein by PARP-1 is conserved PTM implicate in DNA repair, apoptosis, regulation of transcription, anti- recombination, scaffold attachment, and modulation of chromatin structure. PRAP1 directly interact with CENP-A and BUB3 under normal condition. Poly-ADP-ribose location of these proteins upon DNA damage induced by $\mathrm{V}$-irradiation. Poly-ADP-DNA ribosylation of protein changes the activity or function of centromere associated protein [7]. Change in the activity or function of this protein suggests that PRAP-1 is likely to involve many different functions such as kinetochore assembly/ disassembly and spindle checkpoint control.

\section{Post-transcriptional regulation by PARylation of RNA binding protein: -}

When a particular RNA Transcript is synthesized there no guarantee that it will create a functional protein in the cell. All the RNA processing steps are intricately controlled by RNA-binding protein (RBPs), which are associated with pre-mRNA/mRNA during the lifespan of a particular RNA.RBP can be modified at the post transcript level by phosphorylation. And PARylation to achieve temporal post-transcriptional control of gene regulation [5]. PARylation is performed by an enzyme PARP. Poly-ADPribosylation after the physical and enzymatic property of acceptor protein which becomes highly -very charge. PARylation involved in the regulation of many biological processes such as chromatin modulation, transcription control [5]

- Inhibition of phosphorylation of S/R protein by PolyADP-ribose

- ASF/SF2, SF3BI, SF3AI, and SF3B2 are the splicing factor which is associated with (PADPr)

- ASF/SF2 a prototypical serine- arginine-rich protein. PADPr bind ASF/SF2 via either the RPM1 or RS main, but not the RRM2 domain.

- Phosphorylation of the serine residue of ASF/SF2 promote the splicing, binding of pADPrtoAS/SF2 inhibited the phosphorylation of ASF/SF2.

- PDAPrbindingto SR protein modulating phosphorylation pf SR protein and also regulate the alternative splicing.

\section{$>$ Regulation of Ribosomal RNA Processing-}

The functional center for ribosomal DNA transcription and rRNA processing is nucleoli about $40 \%$ of PRAP1 is accumulate in the nucleoli. Various rRNA associate nuclear proteins such as fibrillarin, nucleolin, 
nucleophosmin are associated with poly ADP ribose. Disruption of polyADPr metabolism by either PARP1 and PARG loss of the function causes mislocation of these proteins which results in rRNA processing defect [5].

\section{* Effect of Parylation In S100-Calcium Binding Protein B In Muscle Cell}

$\mathrm{S} 100 \mathrm{~B}$ is also known as $\mathrm{S} 100$ calcium binding protein $\mathrm{B}$, normally seen in the nervous cell; a macromolecule. The intake property of insulin is carried out by glucose to various tissues like myoblast and muscle cell $[8,9]$. Normally while in the respiration process, S100B [10] affects glycolysis by up taking glucose by the ADPRibosylation process. In meanwhile the Ribosylation process effect the glycolytic metabolism [16], lactose production as well as enzymatic activity in myoblast cell and muscle cell. S100B has been demonstrated that on Adipocytes, so it also observed that the serum level of fat or obese mice is less healthy than other mice. By experiment it was stated that the S100B was disturbing glucose uptake effect insulin concentration, while in experiment the glycolysis, which independent of insulin signaling process. By finding, S100B might disturb Glyceraldehyde-3- phosphate dehydrogenase (GAPDH) $[8,17,10]$ in reducing glucose utilization. The impact of S100B to PARylation of GAPDH [18] in immune response. There is no response carried out byS100B in extracellular as well as intracellular; which gives the NADH ratio by PARylation. Some special types of PARP inhibiting 3$\mathrm{ABA}$, which recovered $\mathrm{S} 100 \mathrm{~B}$, involved in glucose utilization. The S100B -PARP interaction may be a core mechanism while the extracellular S100B [13,14] affects the glucose mechanism. So the PolyADPRibo) selection process. RAGE; a receptor, which receives the promoting signaling as NF-kB and Erk whereas RAGE; does not affect S100B in the cell.

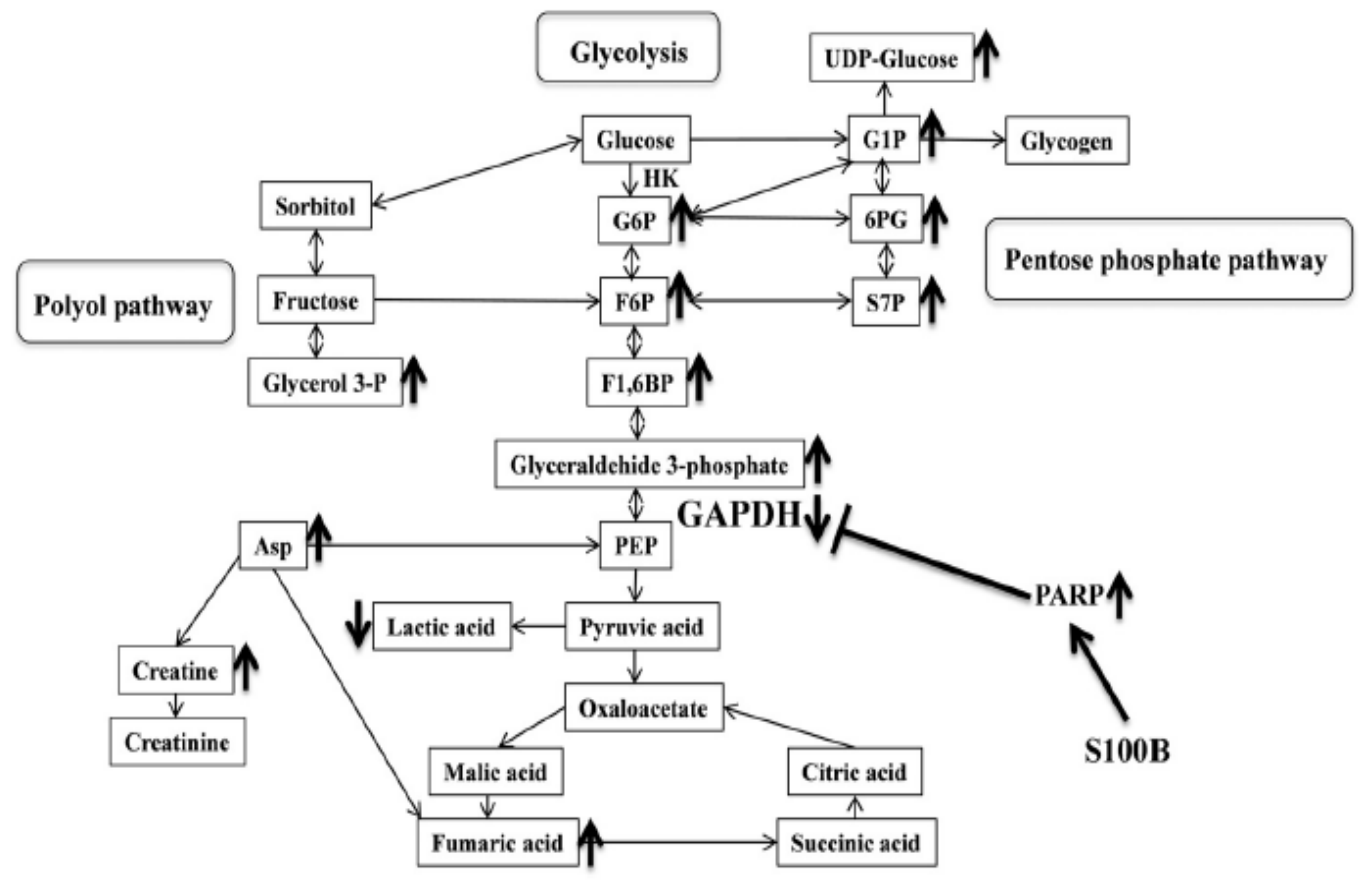

Fig 2:- Schematic diagram of S100B which effect of glucose

In some cases $\mathrm{S} 100 \mathrm{~B}$ effect some signals like TLR, TLR-4 which is largely affected by S100B. In such a case S100B stimulates ERK in myoblast. Oxidative stress enhancing PARP activity, the hydrogen peroxide $\left(\mathrm{H}_{2} \mathrm{O}_{2}\right)$ blocked the glycolysis. By Ribosylation the ratio of GSHGSSG a substitute endpoint of oxidative stress, this was not changed by $\mathrm{S} 100 \mathrm{~B}$ treatment. Other markers like as 4hydroxynonenal and $\mathrm{O}_{2}$ radicals were not changed by S100B. At last, oxidative stress doesn't affect on glycolysis [19].

From the latest study, the extracellular S100B is gathered by endocytic vesicles \& transforming into the cell with $\mathrm{n}$ little short period i.e. $3 \mathrm{~h}$. In conclusion, S100B may prevent glycolysis in muscle cells by suppressing GADPH activity in a cell, which enhanced by PARylation.

\section{PATHOLOGICAL CONDITION}

\section{Cd38 Knockout Mice Show Unique Defense Against} Ischemic Brain Damage In Spite Of High Level Parylation

NAD is required as the essential co-factor in the cellular bioenergetics by several enzymes. The depletion of the NAD following the ischemic insult results in cell decease and is associated with the over activation of the PARP1 as well as NAD consuming enzyme CD38 in an increased level [20]. To regulate the CD38 activity in the post-ischemic brain damage the CD38 knockout mice and wild type mice both were subjected to transient forebrain ischemia. The CD38 is expressed in various cell types and tissue including the hematopoietic derived cells such as monocyte, dendritic cell, lymphocytes, and microglia, also called as the main enzyme that controls cytosolic NAD 
levels [21]. NAD as a substrate uses an enzyme CD38 to generate cyclic ADP ribose. CD38 activity increases brain tissue following ischemic insult and effectively depletes the brain tissue of NAD. It was found that CD38 knockout sham mice express a higher number of CA1 neurons as compared to the wild type group. The wild type mice have $50 \%$ of CA1 neurons survived after ischemic insult [22]. But to the surprise it was seen that CD38 knockout mice resulted in only a $26 \%$ decrease in CA1 surviving neurons when compared to CD38 knockout sham animals which suggested that CD38 contributes to the cell death mechanism [23]. Again, next it was examined whether the CD38 can contribute to post-ischemic NAD degradation and its effect on PARP development. It was seen that wild type animals recovered in $24 \mathrm{hrs}$ with $40 \%$ NAD whereas CD38 knockout mice recovered in $4 \mathrm{hrs}$ after ischemia. Although CD38 is not directly involved in PAR metabolism, the control CD38 knockout animal disclosed $140 \%$ higher PAR levels when compared to wild type animal [24]. Since the poly ADP ribosylation levels are determined by the activity of both PARP1 and PAR catabolizing enzyme poly-ADP-ribose glycohydrolase (PARG), the PARG levels were examined which showed that control sham operates animals the PARG levels were lesser in CD38 knockout mice when it compared to wild type which suggests that PAR hydrolysis was less effective leading to high cellular PARylation levels.
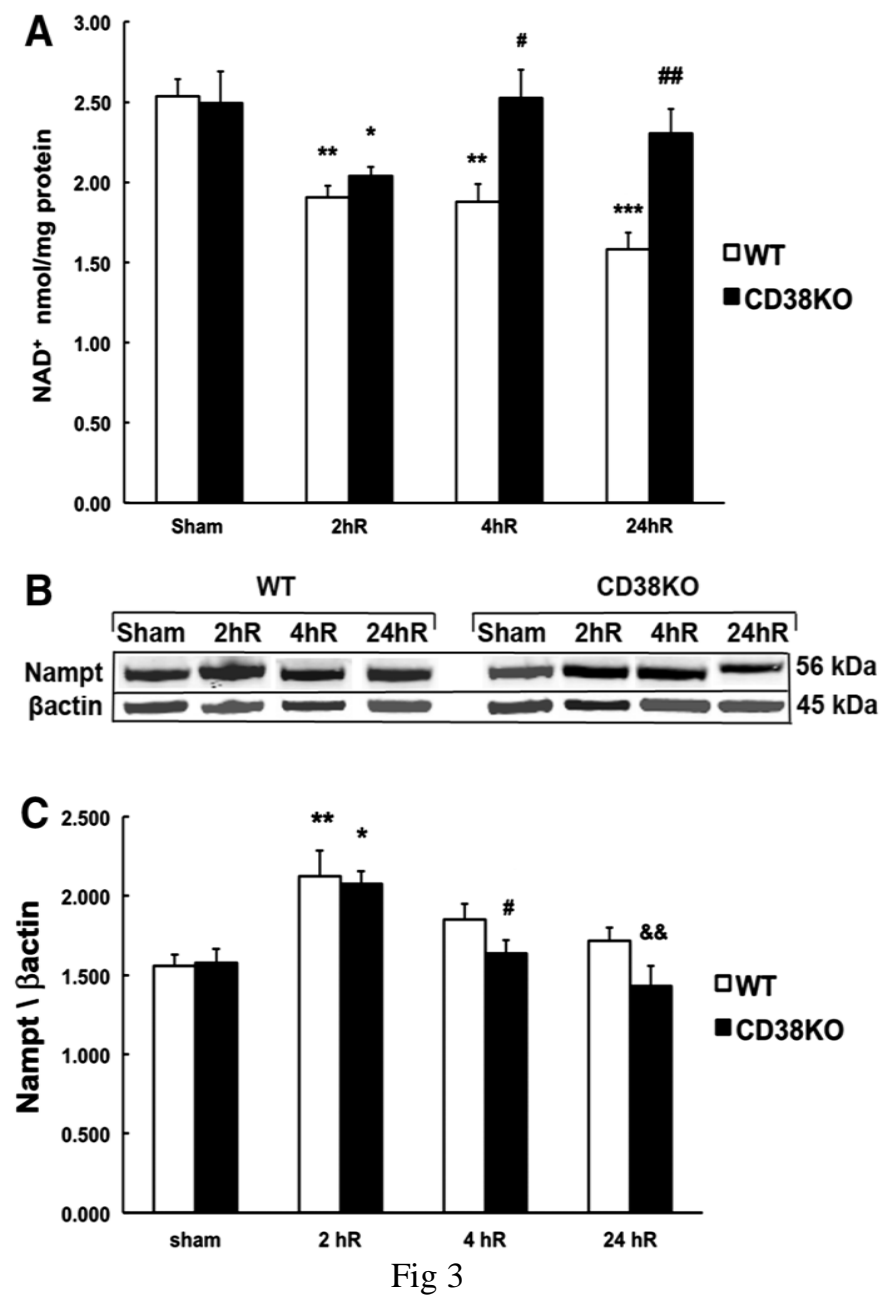

Thus the present study indicates that there is a need to work out carefully when using CD38 knockout animals as a genetic material to study the effects and further the data suggest that disrupting the CD38 activity and suppression of metabolic pathway related directly, but also has a side effect on the expression levels of enzyme take part in NAD metabolism are affected [22]. The CD38 animals showed high levels of PARylation of cytosolic proteins and are more resistant to ischemic brain damage as compared to the wild type animals. Thus, it indicates that PAR accumulation is lethal to cells and the PAR polymer is a decease signal.

\section{Activation Of PARP Contributes To Development Of Doxorubicin-Induced Heart-Failure}

PARP when activated by single-stranded DNA disrupts and initiates an energy-consuming cycle by transferring ADP ribose until it forms NAD to nuclear protein. The PARP over-activation represents a significant mechanism of tissue injury in many pathological conditions which are related with oxidant stress which includes myocardial reperfusion injury, stroke, circulatory shock and the destruction of auto-immune beta cells. Further, the activation of PARP also contributes to the development of cardiovascular dysfunction in diabetics. Thus, doxorubicin is an antibiotic frequently used to treat number of cancers, which includes severe leukemias, lymphomas, and tumor [25]. Here the test shows whether the damage of the cardiac function in doxorubicin which induces acute heart failure is highly dependent upon the activation of the PARP pathway within the heart. At first when the PARP-1+/+ and PARP-1 -/- were treated with doxorubicin, the heart rate, mean blood pressure, left ventricular systolic pressure decreased whereas left ventricular diastolic pressure increased in case of PARP-1 (+/+) [26]. In the case of PARP1 (-/-) there was an unique improvement in the left ventricular performance. Again, the experiment was done to know the effect of PJ34 on doxorubicin-induced cardiac dysfunction. It was found that there were changes in left ventricular systolic pressure, mean blood pressure, and left ventricular and diastolic pressure [27]. Thus, the PARP inhibitor exerted no effect on the hemodynamic parameter in control mice. The results showed that the activation of PARP in the myocardium may contribute to the damaged cardiac function because PARP-1 (-/-) because the animals resulted in the moderate, not remarkable reduction in their activity. Further when treatment with PJ34 there was a significant decrease in the doxorubicin, the overall motility was $76 \%$ and $77 \%$ [28]. Thus, PJ34 is a potent and effective bioavailable PARP inhibitors. And the antioxidant potential of PJ34 cells were analyzed and it was found that it doesn't act as an antioxidant. At last, the useful effects of pharmacology inhibition of PARP with PJ37 in the mouse-model of doxorubicin-induced heart failure also provides us with the evidence that the genetic deletion of the PARP-1 is associated with protein in opposition to doxorubicininduced cardiotoxicity [29]. 

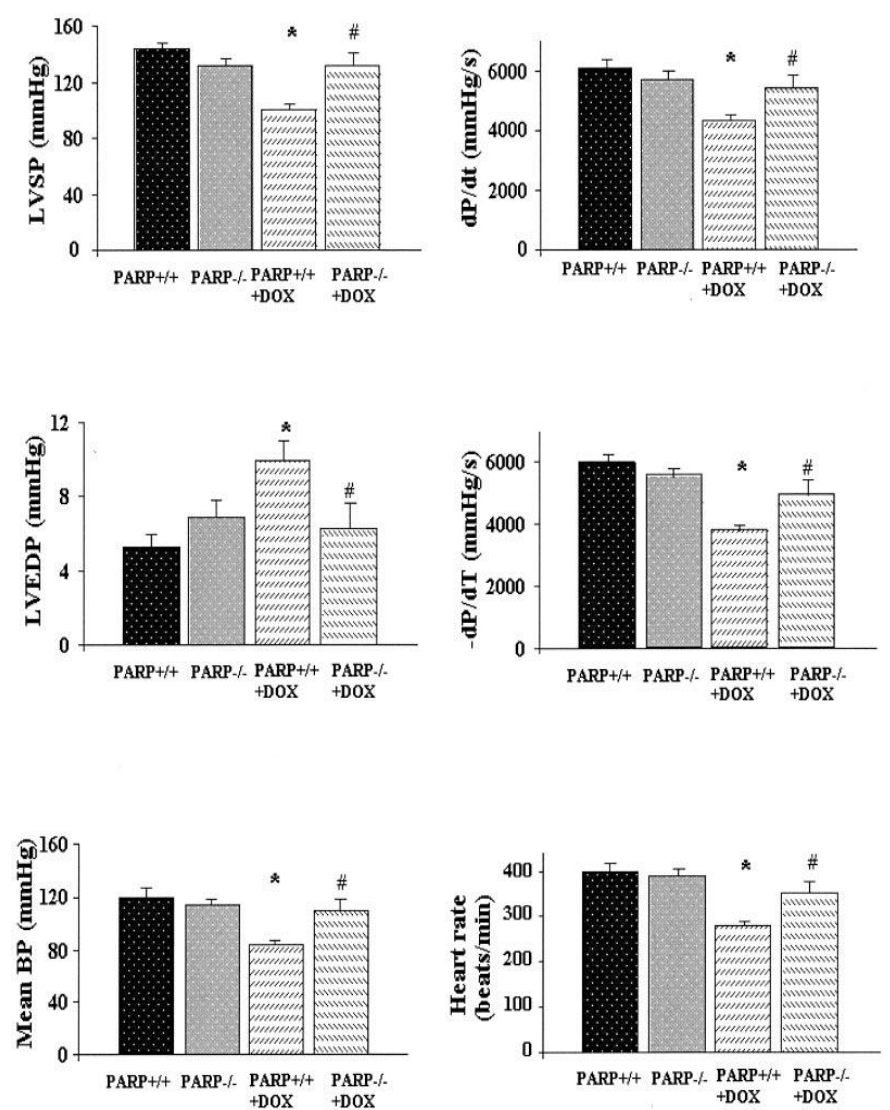

Fig 4

\section{> PARP Inhibitor PJ34 Attenuated Hepatic Triglyceride Accumulation in Alcoholic Fatty Liver Disease in Mice. \\ PARP plays a vital role in transcriptional regulation} and DNA repair and programmed cell death (apoptosis). Alcoholic fatty acid disease (AFLD) is a sever chronic liver disease that occurs due to excess ethanol uptake, which results in the accumulation of lipid in the hepatic cell, increases hepatic lipogenesis and inhibits fatty acid oxidation. Excess uptake of alcohol decreases the hepatic $\mathrm{NAD}^{+}$level [30]. PJ34 [N-(5,6-dihydro-6-oxo-2phenanthridine)-2-acetamide hydrochloride] prevent hepatic PARP activity to regulate the pathological effect on AFLD in mice [31]. As PARP is an $\mathrm{NAD}^{+}$consumer, PARP catalyzes Polymeric adenosine diphosphate ribose (pADPr) synthesis from $\mathrm{NAD}^{+}$, thereby regulating activity excessive hyper-activation of PARP causes the decrease intracellular $\mathrm{NAD}^{+}$and ATP levels, results in disorder or death [32]. For the detection of TG content in hepatic cells, for 4 weeks mice were fed with Lieber-Decarli Alcohol. IT has been observed that due to sever chronic alcohol consumption, results drastic increase in hepatic TG content and circulation of TG levels. Analysis of AFLD by staining results shows that in AF (alcohol-fed -mice) the lobular structure was destroyed and increased lipid found as compared to the PF group (paired-fed mice). Hence collected data proved that sever chronic alcohol consumption results in hepatic lipid accumulation and injury of liver directly [33]. When mice fed with ethanolcontaining liquid diet for 4 weeks results obtained due to chronic alcohol feeding is elevated PRAP expression and activity, which shows greater pADPr attachment with protein as compared to the PF group of mice. Further, reduced hepatic $\mathrm{NAD}^{+}$levels and increase in gene expression and gathering more quantity of protein in TG anabolism such as SREBP-1c, DGAT1, and DGAT-2 as compared to the PF group. Hence the sever-chronic alcohol consumption results in hepatic TG accumulation which in turn activates hepatic PARP overexpression and further hepatic $\mathrm{NAD}^{+}$depletion [34].
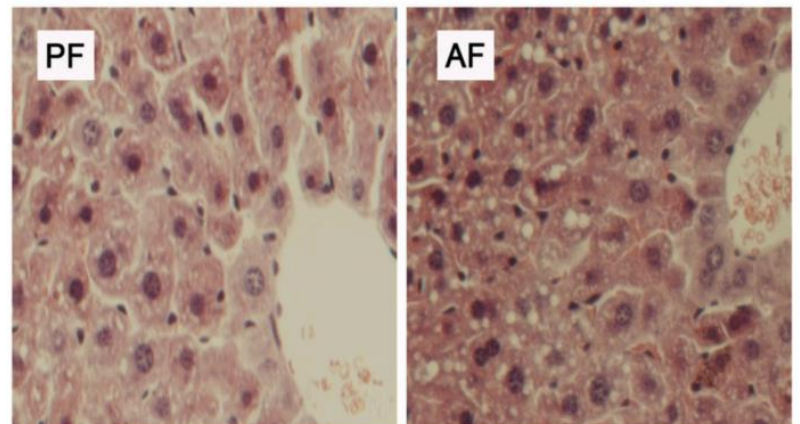

Fig 5

PARP inhibition PJ34 exposure to hepatocytes decreased the pADPr synthesis but did not affect PARP. On PJ34 inhibition of PARP activity significantly increases in NAD+ levels and covered intracellular-TG content occur in hepatic cell. Exposure of hepatocytes to PJ34 the result found that due to PJ34 inhibition significantly lowered the gene expression of DGAT-1 and DGAT-2, but a small change in SERBP-1c is found in PJ34 inhibition of PARP activity suppressed the gene expression in TG anabolism [35]. For determining the PARP inhibition role in the pathogenesis of AFLD mice fed with alcohol containing diet mice with PJ34 injection lowers the hepatic TG content. However, no role of PJ34 injected found in TG content.

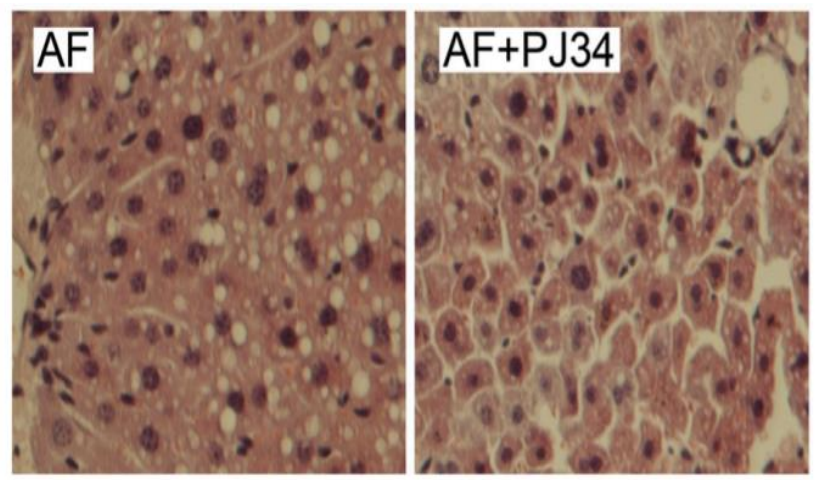

Fig 6

Hence the PJ34 injection attenuated hepatic-TG gathering in AF mice. For checking the effect of PJ34 on the pathogenesis of AFLD mice were fed with alcohol. PJ34 injection suppressed the PARP activation by decreasing the pADPr level as compared to the AF group. Hence result obtained is that PJ34 injection increases TG gathering with high $\mathrm{NAD}^{+}$content and inhibiting TG anabolism in AF mice liver. 


\section{$>$ Role In Neurodegenerative Disorders}

Neurodegenerative disorders eventually are caused by the decease of the neural cells helped by various strange signals. Among the possible modulators of neurodegenerative disorders is the activation of PARylation [45]. Neurodegenerative disease may refer to an array of disorders that are considered as progressive degeneration or decease of nerve cells. Research is being done on the identification of the main effectors and inducers which lead to neurodegeneration, with special attention to the pathways which lead to cell death, including those which are modulated by the accumulation of pADPr by hyperactivity of poly-ADP-ribosylation [46]. Since PARylation is triggered due to intracellular stress along with formation of ROS and DNA-damage, which in turn hyper stimulate PAR synthesis, a typical neurodegeneration. Now it is normally presumed that PAR is a major contributor to the decease or degeneration of neurons which finally causes neurodegenerative disorders. But due to many interdependent routes involved in apoptosis, the role of PAR in the ultimate death of the neuron has not been fully elucidated [47].

Recently, on 18 December 2014, the innovative PARP inhibitor called olaparib was approved for cancer treatment which opens up new dimensions for the development of various type of drug against neurodegeneration triggered by PAR accumulation. However, since PARylation is involved in several elementary processes, these drugs could have negative side effects, thus making a drug to target just a specific group of cells may be difficult [48].

\section{$>$ Preventing Poly-Adp-Ribosylation Increases Fatty Acid Oxidation And Protect Against Fatty Liver Disease:}

Non-alcoholic fatty liver disease (NAFLD) has become a general public health concern and is the most common liver disease in the western world [49]. There is still no approved therapy for NAFLD [50]. PARPs consumes NAD+ for PARylation. It was theorized that over activation of PARPs might lead to the depletion of NAD ${ }^{+}$. Therefore, the inhibition of PARPs was done to check if it is effective in improving hepatic fatty acid oxidization. In mice models C57BL/6J, the fatty liver disease was induced by giving a high-fat high-sucrose (HFHS) diet. This increased the PARylation of proteins by PARPs with reduced $\mathrm{NAD}^{+}$levels and mitochondrial function and content. And in other cases where HFHS diet was supplemented with PARP inhibitor (PARPi) olaparib, the NAFLD was reversed by depletion of $\mathrm{NAD}^{+}$levels and increase in mitochondrial biogenesis and $\beta$ - oxidation in the liver. The PARPi was also helpful in reducing the ER stress, reactive oxygen species, and fibrosis. The other benefits were delaying the hepatic injury. Hence this study demonstrated the positive effects of PARPi in the NAFLD, and can be a form of treatment after further studies and human trials [51].

\section{$>$ PARylation In DNA Damage Response And Cancer Therapy:}

Cells are exposed to many genotoxic stresses every day like endogenous ROS produced from metabolism, exogenous ionizing radiation and ultraviolet radiation. This can cause around $10^{5}$ DNA lesions per day. Continuous DNA damage without repair can cause chromosome rearrangement, mitotic failure, and harmful mutations which can further cause diseases like cancer. Throughout evolution, cells developed very efficient mechanisms to restoration DNA damage and maintain genomic stability. DNA-damage response (DDR) involves processes like DNA damage detection, cell cycle checkpoint regulation, chromatin reorganization, DDR factor recruitment, DNA repair and also processing. PARylation is a critical PTM that initiates DDR and regulates it. In humans, there are 17 protein members in PARP family proteins. When there is DNA damage, especially PARP-1, rapidly identify the single-strand break (SSB) and double-stranded breaks (DSB). After that it immediately start PAR synthesis. It has been identified that PARP-1 can identify both SSBs and DSBs within one second of the breakage of DNA inside cells. PAR chain at the single-strand breaks also recruits other proteins like X-ray cross-complementing protein 1 (XRCC1) which plays one of the most important roles in DNA base excision repair machinery. Poly-ADPribosylation modification is highly dynamic in $\mathrm{s}$ rapidly degrades in vivo with a half-life of 40 s to 60 minutes. Gathering of undigested PAR can activate cell death through parthanatos, storm of apoptosis [53].

Genomic instability is a hallmark of cancer, which is a result of the high accumulation of DNA damage and subsequent mutation can promote tumor development. The most lethal DNA damage is the double-strand breaks. PARP inhibition is cytotoxic in BCRA deficient cells, oral PARPi therapy has become a successfully used for targeted cancer therapy and as a part of standard clinical care for ovarian cancer in gBRCAmut carriers. Results from oral PARPi late phase trails are being greatly anticipated [52].

\section{CONCLUSION}

From the study it is seen that the physiological function includes the DNA-base excision, DNA damage signaling, etc. whereas the pathological effects include diseases like ischemic of various organs, heart failure, liver problem, diabetes. Numerous studies investigations include the formation of PARylation that has been performed in the last some decades. But our understanding of the mechanism leading the signaling of the PARylation and the physiological and pathological importance of the pathways regulated by ADP ribosylation is still understood poorly. Thus, there are still many exciting findings to be discovered in the field of research and the scientific community which showed that ADP ribosylation has been gradually increasing in past years. Researchers are now giving great time and efforts for investigation and developing in new stages, tools and methods to study the process of PARylation, which greatly facilitates further understanding 
of the complexity of the cellular and molecular mechanism which is controlled by ADP ribosylation.

\section{REFERENCES}

[1]. .Ji, Y., \&Tulin, A. V. (2013). Post-transcriptional regulation by poly (ADP-ribosyl) ation of the RNAbinding proteins. International journal of molecular sciences, 14(8), 16168-16183.

[2]. Aksoy P, White TA, Thompson M, Chini EN (2006), Regulation of intracellular levels of NAD: a novel role for CD38, Biochemistry Biophysics Resources Communication, 345:1386-1392.

[3]. Andrabi SA, Umanah GK, Chang C, Stevens DA, Karuppagounder SS, Gagné JP, Poirier GG, Dawson VL, Dawson TM. Poly(ADPribose)polymerasedependent energy depletion occurs through inhibitionof glycolysis. ProcNatlAcadSci USA 111: 10209-10214, 2014. doi:10.1073/pnas.1405158111.

[4]. Balan IS, Fiskum G, Kristian T (2010), Visualization and quantification of $\mathrm{NAD}(\mathrm{H})$ in brain sections by a novel histoenzymaticnitrotetrazolium blue staining technique, Brain Resources, 1316:112-119.

[5]. Baxter P, Chen Y, Xu Y, Swanson RA (2014), Mitochondrial dysfunction induced by nuclear poly(ADP-ribose) polymerase-1: a treatable cause of cell death in stroke, Translational Stroke Resources, 5:136-144.

[6]. Blower, M. D., \&Karpen, G. H. (2001). The role of Drosophila CID in kinetochore formation, cell-cycle progression and heterochromatin interactions. Nature cell biology, 3(8), 730.

[7]. Caleb Dulaney1, Samuel Marcrom1, Jennifer Stanley, Eddy S. Yang .Poly(ADP-ribose) polymerase activity and inhibition in cancer Seminars in Cell \& Developmental Biology 63 (2017) 144-153.

[8]. Creemers EE, Cleutjens JP, Smits JF, and Daemen MJ (2001), Matrix metalloproteinase inhibition after myocardial infarction: a new approach to prevent heart failure? Circulation Resources, 89: 201-210.

[9]. Davide Alessandro Basello, Anna Ivana Scovassi Poly(ADP-ribosylation) and neurodegenerative disorders * Istituto di GeneticaMolecolare CNR, Via Abbiategrasso 207, 27100 Pavia, Italy Mitochondrion 24 (2015) 56-63 .

[10]. deMurcia, G., and Shall, S. (2000) From DNA Damage and Stress Signalling to Cell Death: Poly ADP Ribosylation Reactions, Oxford University Press, New York

[11]. Devalaraja-Narashimha K, Padanilam BJ. PARP-1 inhibits glycolysis in ischemic kidneys. $J \mathrm{Am}$ SocNephrol20: 95-103, 2009. doi:10.1681/ ASN.2008030325.

[12]. Diefenbach J, Burkle A (2005), Introduction to poly (ADP- ribose) metabolism, Cell and molecular life science, 62: 721-730.

[13]. Diefenbach, J., \&Bürkle, A. (2005). Introduction to poly (ADP-ribose) metabolism. Cellular and molecular life sciences: CMLS, 62(7-8), 721-730.
[14]. Donato R, Cannon BR, Sorci G, Riuzzi F, Hsu K, Weber DJ, Geczy CL. Functions of S100 proteins. CurrMol Med 13: 24-57, 2013. doi:10.2174/156652413804486214

[15]. Donato R, Sorci G, Riuzzi F, Arcuri C, Bianchi R, Brozzi F, Tubaro C, Giambanco I. S100B's double life: intracellular regulator and extracellularsignal. BiochimBiophysActa1793: $1008-1022,2009$. doi:10.1016/j.bbamcr.2008.11.009.

[16]. Doroshow JH and Davies KJ (1986), Redox cycling of anthracyclines by cardiac mitochondria. II. Formation of superoxide anion, hydrogen peroxide, and hydroxyl radical, Journal of Biochemical, 261: 3068-3074.

[17]. Du X, Matsumura T, Edelstein D, Rossetti L, Zsengellér Z, Szabó C, Brownlee M. Inhibition of GAPDH activity by poly(ADP-ribose) polymeraseactivates three major pathways of hyperglycemic damage inendothelial cells. J Clin Invest 112: 1049-1057, 2003. doi:10.1172/JCI18127.

[18]. Emma D. Deeks Olaparib: First Global Approval Drugs February 2015, Volume 75, Issue 2, pp 231240

[19]. Francesca Aredia Anna Ivana Scovass Poly(ADPribose): A signaling molecule in different paradigms of cell death. Author links open overlay panelBiochemical Pharmacology, Volume 92, Issue 1, 1 November 2014, Pages 157-163

[20]. Functions of poly(ADP-ribose) polymerase (PARP) in DNA repair, genomic integrity and cell death ZdenkoHerceg, Zhao-Qi Wang*International Agency for Research on Cancer (IARC), 150 cours AlbertThomas, F-69008 Lyon, France Received 1 October 2000; received in revised form 12 November 2000; accepted 1 December 2000 Mutation Research 477 (2001) 97-110

[21]. Gagne JP, Isabelle M, Lo KS, Bourassa S, Hendzel MJ, Dawson VL, Dawson TM, Poirier GG(2008), Proteome- wide identification of poly(ADP-ribose) binding proteins and poly(ADP-ribose)-associated protein complexes, journal of Nucleic acids resources, 36: 6959-6976.

[22]. Gariani K, Menzies KJ, Ryu D, Wegner CJ, Wang X, Ropelle ER, Moullan N, Zhang H, Perino A, Lemos $\mathrm{V}$, et al. (2016), Eliciting the mitochondrial unfolded protein response by nicotinamide adenine dinucleotide repletion reverses fatty liver disease in mice, Hepatology journal, 63:1190-1204.

[23]. Giacco F, Brownlee M. Oxidative stress and diabetic complications. Circ Res 107: 1058-1070, 2010. doi:10.1161/CIRCRESAHA.110.223545.

[24]. Gibson BA, Kraus WL. New insights into the molecular and cellular functions of poly(ADP-ribose) and PARPs. Nat Rev Mol Cell Biol13: 411-424, 2012. doi:10.1038/nrm3376.

[25]. Giovanni Musso, Maurizio Cassader\& Roberto Gambino 
[26]. Hosokawa, K., Hamada, Y., Fujiya, A., Murase, M., Maekawa, R., Niwa, Y., \&Arima, H. (2017). S100B impairs glycolysis via enhanced poly (ADP-ribosyl) ation of glyceraldehyde-3-phosphate dehydrogenase in rodent muscle cells. American Journal of Physiology-Endocrinology and Metabolism, 312(6), E471-E481

[27]. Hu B, Gharaee-Kermani M, Wu Z, Phan SH(2011), Essential role of $\mathrm{MeCP} 2$ in the regulation of myofibroblast differentiation during pulmonary fibrosis, American journal of pathology, 178: 15001508.

[28]. Hu B, Wu Z, Hergert P, A. Henke C, B. Bitterman P and H. Phan S (2013), Regulation of myofibroblast differentiation by poly(ADP-ribose) Polymerase-1, The American Journal of Pathology, 182: 71-82.

[29]. Hu B, Wu Z, Phan SH (2003), Smad3 mediates transforming growth factor beta- induced alphasmooth muscle actin expression, American Journal Respiration Cell Molecular Biology, 29: 397-404.

[30]. Huang S, Zhang B, Chen Y, Liu H, Liu Y, Li X, Bao Z, Song Z, and Wang Z (2018), Poly(ADP-Ribose) Polymerase Inhibitor PJ34 Attenuated Hepatic Triglyceride Accumulation in Alcoholic Fatty Liver Disease in Mice, The journal of pharmacology and experimental therapeutics, 364:452-461.

[31]. Ji Y, Tulin AV(2010), The roles of PARP1 in gene control and cell differentiation, Current Opinion in Genetics and Devlopment, 20: 512-518.

[32]. Ji, Y., \&Tulin, A. V. (2013). Post-transcriptional regulation by poly (ADP-ribosyl) ation of the RNAbinding proteins. International journal of molecular sciences, 14(8), 16168-16183.

[33]. Karim Gariani1, Dongryeol Ryu1,y, Keir J. Menzies1,3,y, Hyon-Seung Yi5, Sokrates Stein2,4, Hongbo Zhang1, Alessia Perino2, Vera Lemos2, Elena Katsyuba1, Pooja Jha1, Sandrine Vijgen7, Laura Rubbia-Brandt7, Yong Kyung Kim5, Jung Tae Kim6, Koon Soon Kim5, Minho Shong5, Kristina Schoonjans2, Johan Auwerx1Inhibiting poly ADPribosylation increases fatty acid oxidation and protects against fatty liver disease, Journal of hepatology.

[34]. Kraus WL and Hottiger MO (2013), PARP-1 and gene regulation: progress and puzzles, Molecular Aspects Medical, 34:1109-1123.

[35]. Kristian T, Balan I, Schuh R, Onken M (2011), Mitochondrial dysfunction and nicotinamide dinucleotide catabolism as mechanisms of cell death and promising targets for neuroprotection, J Neuroscience Resources, 89:1946-1955.

[36]. Kurinami H, Shimamura M, Ma T, Qian L, Koizumi K, Park L, Klann E, Manfredi G, Iadecola C, Zhou P (2014), Prohibitin viral gene transfer protects hippocampal CA1 neurons from ischemia and ameliorates postischemic hippocampal dysfunction, Stroke journal, 45: 1131-1138.

[37]. Lasi_E, Galland F, Vardjan N, Šribar J, Križaj I, Leite MC, Zorec R, Stenovec M. Time-dependent uptake and trafficking of vesicles capturingextracellular S100B in cultured rat astrocytes. J Neurochem139: 309-323, 2016. doi:10.1111/jnc. 13754

[38]. Li T and Singal PK (2000), Adriamycin-induced early changes in myocardial antioxidant enzymes and their modulation by probucol, Circulation journal, 102:2105-2110.

[39]. Lívero FA and Acco A (2016), Molecular basis of alcoholic fatty liver disease: from incidence to treatment, Hepatol Resources, 46:111-123.

[40]. Lonn P, van der Heide LP, Dahl M, Hellman U, Heidin CH, MoustakasA(2010), PARP-1 attenuates Smad-mediated transcription, Molecular cell, 40: 521532.

[41]. Mukhopadhyay P, Horváth B, Rajesh M, Varga Z V, Gariani K, Ryu D, Cao Z, Holovac E, Park O, Zhou Z, et al. (2017), PARP inhibition protects against alcoholic and non- alcoholic steatohepatitis, Journal Hepatology, 66:589-600.

[42]. Nishiyama H, Knopfel T, Endo S, Itohara S. Glial protein S100B modulates long-term neuronal synaptic plasticity. ProcNatlAcadSci USA 99: 4037-4042, 2002. doi:10.1073/pnas.052020999.

[43]. Non-alcoholic steatohepatitis: emerging molecular targets and therapeutic strategies Nature Reviews Drug Discovery volume15, pages249-274 (2016)

[44]. Rappou E, Jukarainen S, Rinnankoski-Tuikka R, Kaye S, Heinonen S, Hakkarainen A, Lundbom J, Lundbom N, Saunavaara V, Rissanen A, et al. (2016), Weight loss is associated with increased NAD(1)/SIRT1 expression but reduced PARP activity in white adipose tissue, Journal Clinical Endocrinology Metabolism, 101:1263-1273.

[45]. Saffery, R., Irvine, D. V., Griffiths, B., Kalitsis, P., Wordeman, L., \&Choo, K. A. (2000). Human centromeres and neocentromeres show identical distribution patterns of $>20$ functionally important kinetochore-associated proteins. Human Molecular Genetics, 9(2), 175-185.

[46]. Saxena, A., Saffery, R., Wong, L. H., Kalitsis, P., \&Choo, K. A. (2002). Centromere proteins Cenpa, Cenpb, and Bub3 interact with poly (ADP-ribose) polymerase-1 protein and are poly (ADP-ribosyl) ated. Journal of Biological Chemistry, 277(30), 26921-26926.

[47]. Schreiber V, Dantzer F, Ame JC, de Murica G (2006), Poly(ADP-ribose): novel functions for an old molecule, Nat Rev Mol Cell Biol, 7: 517-528.

[48]. Singal PK and Iliskovic N (1998), Doxorubicininduced cardiomyopathy, National English Journal Medical, 339: 900-905.

[49]. Su, Z., Deshpande, V., James, D. E., \&Stöckli, J. (2018). Tankyrase modulates insulin sensitivity in skeletal muscle cells by regulating the stability of GLUT4 vesicle proteins. Journal of Biological Chemistry, jbc-RA117.

[50]. Virág L. Structure and function of poly(ADP-ribose) polymerase-1: role in oxidative stress-related pathologies. CurrVascPharmacol3: 209-214, 2005. doi:10.2174/1570161054368625. 
[51]. Wei-Hsien Houa, Shih-Hsun Chenb, Xiaochun Yub Poly-ADP ribosylation in DNA damage response and cancer therapy,* Mutation Research-Reviews in Mutation Research

[52]. Wong RJ, Aguilar M, Cheung R, Perumpail RB, Harrison SA, Younossi ZM, Ahmed A Nonalcoholic steatohepatitis is the second leading etiology of liver disease among adults awaiting liver transplantation in the United States. Gastroenterology. 2015 Mar;148(3):547-55.

[53]. Xu MF, Tang PL, Qian ZM, and Ashraf M (2001), Effects by doxorubicin on the myocardium are mediated by oxygen free radicals, Life Science journal, 68: 889-901.

[54].ZdenkoHerceg, Zhao-Qi Wang Functions of poly(ADP-ribose) polymerase (PARP) in DNA repair, genomic integrity and cell death *International Agency for Research on Cancer (IARC), 150 cours Albert-Thomas, F-69008 Lyon, France Received 1 October 2000; received in revised form 12 November 2000; accepted 1 December 2000 Mutation 